\title{
Alumnos en pandemia: una mirada desde el aprendizaje autónomo
}

\author{
Larisa Enríquez Vázquez y Myrna Hernández Gutiérrez
}

\begin{abstract}
Resumen
El aprendizaje autónomo es un concepto que refiere a la formación de los estudiantes como aprendices, con la capacidad de tomar el control sobre su propio proceso de aprendizaje, para el resto de la vida. El año 2020 ha sido determinante para mostrar la necesidad y urgencia de fomentar habilidades que permitan a los estudiantes ser autogestivos, y regular sus procesos de aprendizaje. En este contexto emergente actual, es necesario preguntarnos, como docentes, cómo apoyamos a los estudiantes en el fomento de estas habilidades, cuáles son las prioritarias y cuáles las que se tendrían que impulsar, ya sea porque existe una deficiencia en ellas o un mayor interés entre los jóvenes, y cómo podemos ayudar a los alumnos en la mejora de su proceso de aprendizaje.

Con la intención de generar opciones viables en el marco de la pandemia de la covid-19, se inició una investigación sobre el nivel de autonomía que tienen los estudiantes en México. Para ello, durante el mes de mayo de 2020 se aplicó el "Cuestionario aprendizaje autónomo en tiempos de coviD-19". Este trabajo presenta los resultados de dicho sondeo.
\end{abstract}

Palabras clave: aprendizaje autónomo, pandemia, evaluación del aprendizaje autónomo.

\section{StUdeNTS IN PANDEMIC: A GLIMPSE FROM AUTONOMOUS LEARNING}

\begin{abstract}
Autonomous learning is a concept that refers to training students as lifelong learners, with the ability to take control over their own learning process. The year 2020 has shown the need and urgency to promote skills that allow students to be self-managed and regulate their learning processes, including the development of skills to plan their activities and self-evaluate. In this current emerging context, it is necessary to ask ourselves, as teachers, how can we support students in fostering these skills, which of these should be considered as priorities and which ones should be promoted, either because there is a deficiency in them or a greater interest among young people, and how can we, as teachers, help students in its training.

With the objective of generating viable options in the framework of the coviD-19 pandemic, an investigation was initiated on the students' level of autonomy in Mexico. To do this, during May 2020 we applied the "Autonomous learning survey in times of covid-19". This work presents the results of such exploration.
\end{abstract}

Keywords: autonomous learning, pandemic, evaluation of autonomous learning.

Recepción: 7/10/2020. Aprobación: 9/02/2021.

Dol: http://doi.org/10.22201/cuaieed.16076079e.2021.22.2.11

Universidad Nacional Autónoma de México, Coordinación de Universidad Abierta, Innovación Educativa y Educación a Distancia (CUAIEED) Este es un artículo de acceso abierto bajo la licencia de Creative Commons 4.0 (c) (1)(2) 
"Alumnos en pandemia: una mirada desde el aprendizaje autónomo" Larisa Enríquez Vázquez y Myrna Hernández Gutiérrez Vol. 22, Núm. 2, marzo-abril 2021 Revista Digital Universitaria

\section{Larisa Enríquez Vázquez}

larisa_enriquez@cuaieed.unam.mx orcid.org/0000-0003-3587-4253

Maestra en ciencias por la Universidad Nacional Autónoma de México (UnAM)y doctorante en e-learning por la Universidad Abierta de Cataluña. Investigadora de tiempo completo en la Coordinación de Universidad Abierta, Innovación Educativa y Educación a Distancia (CUAIEED) de la unAm. Sus líneas de investigación son el aprendizaje autónomo, modelos flexibles para la educación, y educación abierta y a distancia.

\section{Myrna Hernández Gutiérrez}

myrna_hernandez@cuaieed.unam.mx orcid.org/0000-0002-0485-9037

Licenciada en Informática por la UNAM, maestra en Comunicación y Tecnologías Educativas por el Instituto Latinoamericano de la Comunicación Educativa (ILCE) y doctora en Pedagogía por la uNAM. ES académica en la Coordinación de Universidad Abierta, Innovación Educativa y Educación a Distancia (CUAIEED) de la unam. Su línea de investigación es el aprendizaje colaborativo en ambientes virtuales. 
"Alumnos en pandemia: una mirada desde el aprendizaje autónomo" Larisa Enríquez Vázquez y Myrna Hernández Gutiérrez

Vol. 22, Núm. 2, marzo-abril 2021

Revista Digital Universitaria

\section{Introducción}

El aprendizaje autónomo es un concepto que ha ido tomando mayor fuerza e interés en el ámbito educativo. La autonomía del aprendizaje, como indican Rodríguez, Hernández y Dávalos (2018), se refiere a que el alumno se gobierne a sí mismo, y que pueda tomar decisiones sobre su aprendizaje, gracias al entrenamiento y desarrollo de competencias o habilidades cognitivas, afectivas, interactivas y metacognitivas. Otros autores, como Tassinari (2012), indican que la autonomía del alumno es la meta-capacidad para tomar el control del proceso propio, en diferentes grados y de diferentes maneras, según la situación de aprendizaje.

Si se considera que la autonomía es uno de los componentes para aprender a aprender, la función de formar a los estudiantes como aprendices para el resto de la vida, con la capacidad de controlar su propio proceso de aprendizaje, se vuelve indispensable. Así, el papel del docente y el impacto que puede lograr en el desarrollo de la autonomía para aprender es relevante. Señalan Hernández et al. que el rol del docente es incentivar y provocar, ser acompañante, tutor y guía del alumno, ayudándole a descubrir sus capacidades, recursos y destrezas (2018, p. 350).

El año 2020 ha mostrado la necesidad y urgencia de fomentar habilidades que permitan a los estudiantes ser autogestivos y autoregulados, incluyendo además competencias para planear sus actividades de aprendizaje y autoevaluarse. En este sentido, durante el mes de mayo de 2020 se aplicó el "Cuestionario aprendizaje autónomo en tiempos de covid-19", con la intención de identificar el nivel de autonomía que tienen los estudiantes en México. Este instrumento se realizó con base en el modelo de Tassinari, e incluye las cinco dimensiones que contempla el aprendizaje autónomo según la autora. Las dimensiones evaluadas fueron las siguientes:

- Afectiva. Vinculada con los sentimientos, emociones, voluntad y motivación que poseemos para aprender algo.

- Social. Es el aprendizaje y la negociación del aprendizaje que logramos a partir de la interacción con pares, asesores, profesores, entre otros.

- Cognitiva. Referente al conocimiento y las estrategias que empleamos para avanzar en el logro de una actividad u objetivo.

- Metacognitiva. Sobre la conciencia y conocimiento que poseemos sobre el proceso que empleamos para aprender algo.

- Orientada a la acción. Considera el comportamiento, habilidades, actitudes y toma de decisiones que ponemos en práctica al momento de aprender algo. 


\section{Sobre el cuestionario}

El cuestionario se aplicó vía web. Lo respondieron 725 estudiantes, de ellos sólo 574 contestaron el total de 20 preguntas. Suponemos que algunos alumnos fueron dejando de responder debido a la extensión, y a que sintieron que se repetían preguntas.

En cada bloque se incluyó un espacio para agregar comentarios relacionados con las preguntas y la dimensión en cuestión. A partir del análisis de los resultados cuantitativos y los cualitativos, tenemos consideraciones para cada una de las dimensiones.

\section{Datos sociodemográficos}

Hubo representación de personas de diferentes niveles educativos, que viven en 20 estados de la República. 67\% vive en la zona metropolitana del valle de México (ver video 1).

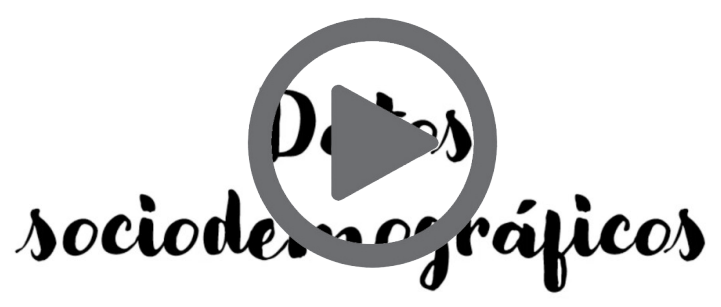

Video 1. Datos sociodemográficos.

\section{Dimensión afectiva}

Las preguntas están divididas en dos bloques. El primero corresponde a emociones y sentimientos involucrados al aprender algo. El segundo, al sentimiento de aprender en la modalidad a distancia.

En el primer bloque, 48\% señaló conocer y controlar la motivación que tiene para aprender, mientras que 50\% indicó que intenta elevar la motivación por el estudio cuando ésta decae. Además, 21\% y 25\% de los estudiantes mencionaron querer aprender a convertir sentimientos negativos en positivos y hablar de la manera cómo se sienten durante el proceso de aprendizaje, respectivamente.

En el segundo bloque, $40 \%$ de los alumnos pudo identificar claramente cómo se siente aprendiendo con el uso de las tecnologías y $47 \%$ indica que logra 
"Alumnos en pandemia: una mirada desde el aprendizaje autónomo"

Larisa Enríquez Vázquez y Myrna Hernández Gutiérrez

Vol. 22, Núm. 2, marzo-abril 2021

Revista Digital Universitaria

buscar los canales de comunicación con los que se siente cómodo para interactuar. En contraste, casi $17 \%$ de los alumnos señala querer aprender a pedir apoyo para desenvolverse mejor en un ambiente digital.

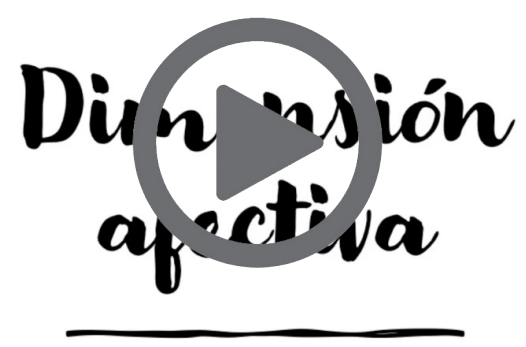

Video 2. Dimensión afectiva.

En lo referente a los aspectos cualitativos, los estudiantes indican que éstos impactan su motivación para estudiar. Existen casos de alumnos que no saben manejar sus emociones y sentimientos, y también mencionan el no tener una persona con la cual hablar. En la situación de aprendizaje en el nuevo entorno digital, diversos alumnos mencionan, no sólo depresión por la situación de confinamiento y enfermedad, sino también desánimo y frustración porque los profesores no responden sus mensajes ni ayudan a aclarar dudas. Parece ser que los docentes no están cubriendo las expectativas en cuanto a la comunicación en el proceso de enseñanza-aprendizaje y, en opinión de los alumnos, tampoco los están apoyando para afrontar emocionalmente la pandemia.

\section{Dimensión social}

Las preguntas se enfocaron en el estudio a distancia. El primer bloque integra preguntas relacionadas con la intención de buscar ayuda y apoyo en otros para entender un tema o estudiar juntos, mientras que el segundo se refiere a las habilidades y actitudes que ejerce el estudiante ante aquellas actividades que se realizan en grupo.

En el primer bloque, 50\% de los alumnos manifestó que puede localizar a alguien que les aclare una duda o explique un tema. La actividad que más les interesa (14\%) aprender es el decidir cuándo es el mejor momento para trabajar o completar una tarea con la ayuda de otros. 6\% de los estudiantes considera que no es importante tener un compañero o equipo con quien estudiar.

En el segundo bloque, cuando hay debates y negociación de ideas, 77\% de los alumnos indicó que puede escuchar las posturas de los otros, aunque sólo 39\% intenta expresar sus propias posturas y 13\% quisiera aprender a expresarlas. Para 6\% no es importante la actividad de comparar su postura con la de otros. 
Video 3. Dimensión social.

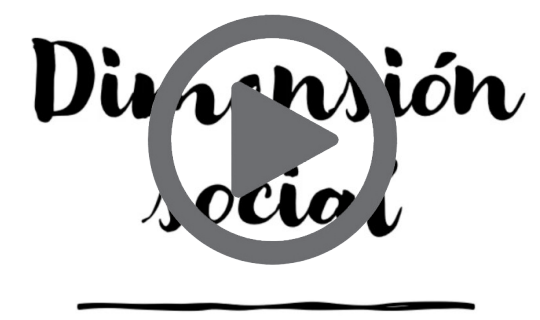

En lo cualitativo, se destacan los aspectos que impactan en el trabajo en equipo y la colaboración. Hay un porcentaje de alumnos (6\%) que no considera importante tener un compañero o un equipo con quien estudiar, así como tampoco le interesa comparar sus posturas con las de otros. Llama la atención que existan algunos comentarios relacionados con la poca credibilidad que los estudiantes le dan a otras personas que no sean los profesores y que por ello no existe la confianza para trabajar con compañeros. Así, el reto para los docentes está en ayudar a los alumnos a identificar los momentos y los canales por los cuales pueden pedir apoyo. El profesor tiene una participación fundamental en el cambio de paradigma de un aprendizaje individual y en solitario hacia un aprendizaje con y de otros, lo que implica una valoración positiva del conocimiento que tiene cada individuo.

\section{Dimensión cognitiva}

Está centrada en las habilidades de control y gestión de una tarea de aprendizaje. Implica habilidades de planeación, selección de métodos y estrategias, y evaluación de los resultados obtenidos, para una actividad concreta. Aquí se incluyen tres bloques de preguntas.

El primero considera preguntas relacionadas con la valoración de la tarea o actividad de aprendizaje a realizar. De entre los estudiantes, 68\% indica poder determinar si la tarea es fácil o difícil. Sobre la posibilidad de identificar actividades adicionales que ayudarán a cumplir con la tarea, 42\% de los alumnos indica que intenta hacer dicho reconocimiento, mientras que 13\% quisiera aprender a reconocer otras actividades.

Para el bloque relacionado con la valoración del progreso obtenido, $54 \%$ indica que puede reconocer si los materiales y los recursos empleados fueron adecuados para su nivel de conocimiento. De la muestra, 46\% intenta valorar si los métodos y las estrategias utilizadas son acordes con el aprendizaje esperado, mientras que 6\% señala que le gustaría aprender a llevar a cabo ese reconocimiento. 
"Alumnos en pandemia: una mirada desde el aprendizaje autónomo" Larisa Enríquez Vázquez y Myrna Hernández Gutiérrez

Vol. 22, Núm. 2, marzo-abril 2021

Revista Digital Universitaria

En el tercer bloque, sobre la evaluación del progreso, 57\% de los alumnos señala que pueden hacer dicha valoración con la ayuda del profesor, 35\% intenta hacerlo con la ayuda de otros (no necesariamente el profesor) y 17\% indica que le gustaría aprender a hacer la evaluación con listas de cotejo.

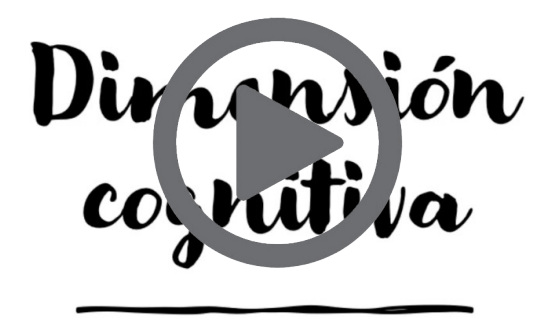

Video 4. Dimensión cognitiva.
En el aspecto cualitativo, según comentarios concretos, el apoyo que requieren los alumnos de los docentes se centra en las habilidades para reconocer actividades adicionales, que apoyen y contribuyan a la realización de tareas, así como para identificar estrategias y métodos para realizarlas, y para poder hacer evaluaciones entre pares. Por ello, es fundamental que el docente oriente su práctica a enseñar técnicas a los estudiantes, así como estrategias y herramientas que puedan utilizar en el desarrollo de tareas, distinguiendo las posibilidades y limitaciones de cada una.

\section{Dimensión metacognitiva}

Está vinculada con la conciencia y control del proceso de aprendizaje que emplean los estudiantes en general. En el cuestionario, existe un bloque relacionado con el establecimiento de metas de aprendizaje, otro con la planeación del aprendizaje, uno más sobre la selección de estrategias y métodos de estudio, y un último bloque que refiere a la autoevaluación de las decisiones tomadas.

Relacionado con las metas, 69\% señala poder establecerlas, teniendo en cuenta sus necesidades e intereses personales. Asimismo, 43\% indica que intenta establecer las metas de acuerdo con los objetivos de aprendizaje de un curso, mientras que 9\% quisiera poder establecerlas de acuerdo con los objetivos del curso, y 8\% quisiera aprender a establecer metas, de acuerdo con las condiciones bajo las cuales deben de estudiar.

En cuanto a las acciones y decisiones que toma un alumno para alcanzar las metas de aprendizaje, 54\% indica que puede hacer un plan de trabajo, mientras que $43 \%$ intenta organizar su tiempo adecuadamente, evaluar sus avances y 
"Alumnos en pandemia: una mirada desde el aprendizaje autónomo" Larisa Enríquez Vázquez y Myrna Hernández Gutiérrez

Vol. 22, Núm. 2, marzo-abril 2021

Revista Digital Universitaria

logros progresivos y alcanzar dichas metas. Por último, 18\% indica que quisiera aprender a evaluar los logros y avances que va obteniendo.

Sobre los métodos y estrategias que utilizan para alcanzar metas de aprendizaje, 51\% de los alumnos señala que puede analizar si son factibles dichos métodos y estrategias, y $44 \%$ intenta evaluar si son adecuados para el tipo de contenidos que están estudiando. Hay un porcentaje que dice requerir la asesoría de alguien para la selección, y el análisis de los métodos y estrategias de estudio que emplea (13\%).

En lo referente a la conciencia y autoevaluación, 64\% de los alumnos indica que puede identificar cuando alcanza sus objetivos de aprendizaje y $50 \%$ intenta valorar si pudo ceñirse o no al plan de aprendizaje previamente planteado, mientras que 12\% quisiera aprender a hacer esta valoración.

\section{Dirgansión
metacogitiva}

Video 5. Dimensión metacognitiva.

En lo cualitativo, acerca de la conciencia y control del proceso de aprendizaje que emplean los estudiantes, éstos incluyen acciones que van desde la identificación y definición de metas de aprendizaje, hasta la evaluación del desempeño y logros obtenidos, no sólo al final del proceso, sino también durante el mismo, y es precisamente en la evaluación donde se identifican mayores problemas. Sería importante que el docente realizara un diseño didáctico que integrara autoevaluaciones progresivas y continuas, que ayuden a los alumnos a adquirir hábitos y estrategias de planeación, autogestión y autoevaluación.

\section{Dimensión orientada a la acción}

Está relacionada con la toma de decisiones y ejercicios reflexivos que realiza el alumno para encauzar su aprendizaje. Las preguntas están divididas en tres bloques. En el primero, 58\% de los alumnos menciona poder establecer, por sí mismo, sus metas de aprendizaje, mientras que 40\% lo puede hacer con ayuda de otros. A 17\% le gustaría poderlo hacer con ayuda del asesor o profesor. 
Al momento de realizar una tarea, 61\% indica que puede identificar cuál es el objetivo de la misma y $37 \%$ intenta identificar si requiere aprender algo de manera previa, para poder desarrollar la tarea.

Sobre la reflexión de las estrategias empleadas, 37\% de los alumnos intenta valorar si las estrategias utilizadas sirvieron para alcanzar el objetivo de aprendizaje, y $6 \%$ quisiera aprender a valorar si sirvieron para realizar una tarea específica.

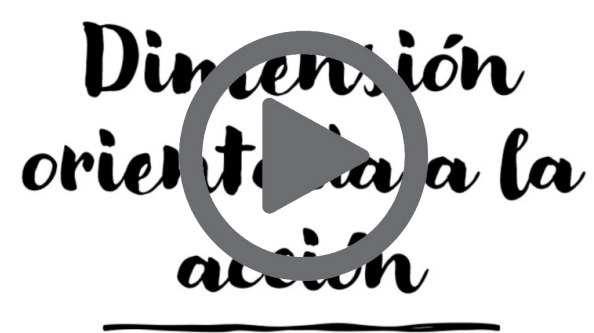

Video 6. Dimensión orientada a la acción.
Si bien los alumnos mencionan la importancia de hacer una planeación, buscar apoyo, del trabajo colaborativo y de organizar el tiempo, el aspecto más relevante es el referente al fortalecimiento de la responsabilidad de los estudiantes con su propio aprendizaje, donde ellos deben involucrarse de manera más profunda en las tareas antes mencionadas. La conciencia del estudiante sobre el beneficio que obtiene con el logro de aprendizaje, acerca de mejorar su propio proceso, y del impacto que esto tiene en los diferentes ámbitos de su vida es una tarea en la que el docente tiene gran participación.

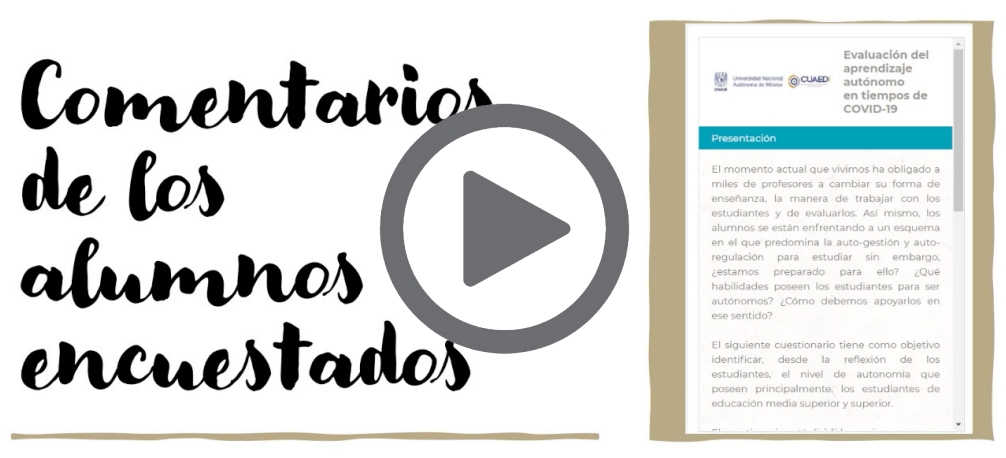

Video 7. Comentarios de los alumnos. 
"Alumnos en pandemia: una mirada desde el aprendizaje autónomo" Larisa Enríquez Vázquez y Myrna Hernández Gutiérrez

Vol. 22, Núm. 2, marzo-abril 2021

Revista Digital Universitaria

\section{Discusión}

La sociedad en el contexto actual tiene como reto un sustancial cambio educativo, para formar ciudadanos autónomos y críticos, que puedan ser aprendices a lo largo de la vida (Ehlers y Kellermann, 2019). Si bien las propuestas didácticas realizadas a partir de metodologías centradas en el alumno promueven el desarrollo de la autonomía, es fundamental resaltar el papel del docente en este tránsito, que se preocupe por crear experiencias formativas basadas en las características, necesidades e intereses del individuo que aprende. Un docente que impulse la participación del alumno y lo oriente para que conecte el aprendizaje con sus intereses, y para que construya redes de aprendizaje y seleccione métodos para autoevaluarse (Cobo, 2016).

Por ello, consideramos que, a partir de esta evaluación, es clara la participación que debería tener el docente en, además de la tradicional dimensión cognitiva, las dimensiones metacognitiva, social, y la orientada a la acción. Pero, sobre todo, en el contexto generado por la covid-19, en la dimensión afectiva-emocional, ya que la emergencia actual evidencia la necesidad de acompañamiento y escucha.

\section{Referencias}

* Cobo, C. (2016). La Innovación Pendiente. Reflexiones (y Provocaciones) sobre educación, tecnología y conocimiento. Colección Fundación Ceibal; Debate.

* Ehlers, U. D. y Kellermann, S. (2019). Future Skills - The Future of Learning and Higher education. Results of the International Future Skills Delphi Survey. https:// www.researchgate.net/publication/332028491_Future_Skills_-_The_Future_of_ Learning_and_Higher_education_Results_of_the_International_Future_Skills_ Delphi_Survey

* Rodríguez Morúa, G., Hernández V., A. L. y Dávalos Osorio, V. (2018, marzo). Autonomía del aprendizaje y pensamiento crítico. En J.C. Martínez (Coord.), III Congreso online internacional sobre la educación en el sig/o XXI (pags. 348351). Universidad de Málaga. https://www.eumed.net/actas/18/educacion/29autonomia-del-aprendizaje-y-pensamiento-critico.pdf

* Tassinari, M. G. (2012). Evaluating Learner Autonomy: A Dynamic Model with Descriptors. Studies in Self-Access Learning Journal, 3(1), 24-40. https://sisaljournal. org/archives/march12/tassinari/

\section{Cómo CITAR ESTe ARTículo}

* Enríquez Vázquez, Larisa y Hernández Gutiérrez, Myrna. (2021, marzoabril). Alumnos en pandemia: una mirada desde el aprendizaje autónomo. Revista Digital Universitaria (RDU), 22(2). Dol: http://doi.org/10.22201/ cuaieed.16076079e.2021.22.2.11 